\title{
Early Unfavorable Non-Hodgkin Lymphoma
}

National Cancer Institute

\section{Source}

National Cancer Institute. Early Unfavorable Non-Hodgkin Lymphoma. NCI Thesaurus.

Code C53282.

A clinical stage I or II non-Hodgkin lymphoma with at least one of the following risk factors: large mediastinal mass, extranodal spread, elevated erythrocyte sedimentation rate, involvement of at least three lymph node areas, and B symptoms. 